\title{
Safety and Efficacy of Bleb Needling with Antimetabolite after Trabeculectomy Failure in Glaucoma Patients: A Systemic Review and Meta-Analysis
}

\author{
Xuhao Chen $\mathbb{D}^{1,2}$ Lingge Suo, ${ }^{1,2}$ Ying Hong $\mathbb{D}^{1,2}$ and Chun Zhang ${ }^{1,2}$ \\ ${ }^{1}$ Department of Ophthalmology, Peking University Third Hospital, Beijing, China \\ ${ }^{2}$ Beijing Key Laboratory of Restoration of Damaged Ocular Nerve, Peking University Third Hospital, Beijing, China \\ Correspondence should be addressed to Ying Hong; drhongying@bjmu.edu.cn
}

Received 20 June 2020; Revised 11 October 2020; Accepted 23 October 2020; Published 30 November 2020

Academic Editor: Roberto dell Omo

Copyright (c) 2020 Xuhao Chen et al. This is an open access article distributed under the Creative Commons Attribution License, which permits unrestricted use, distribution, and reproduction in any medium, provided the original work is properly cited.

\begin{abstract}
Background. Bleb needling with subconjunctival injection of antimetabolites had become a widely accepted approach for trabeculectomy failure. However, IOP reduction effects, success rates, and complications occurrence for this procedure showed great inconsistency among the different studies. Methods. We conducted a literature search on PubMed, Embase, Cochrane Library, and ClinicalTrials.gov. A random-effects model was performed on the extracted data based on the included studies. The intraocular pressure (IOP) and number of antiglaucomatous medications before and after the surgery were pooled for meta-analysis. The success and complication rates were estimated based on the results. Subgroup analysis, sensitivity analysis, and metaregression were applied to explore the origination of heterogeneity. Results. Thirty-seven studies with a total of 2182 patients were finally included in our review. For the present meta-analysis, the overall effects of bleb needling at the last visit revealed a reduction in IOP of $9.74 \mathrm{mmHg}$ (95\% confidence interval (CI) [8.85, 10.63]), 45.9\% (95\% CI [39.0\%, 53.0\%]) for complete success rate, and $70.4 \%$ (95\% CI $[63.5 \%, 77.0 \%])$ for qualified success rate. Application of mitomycin C (MMC) and 5-fluorouracil (5-Fu) during the procedure were efficacious for IOP control during the follow-up. Metaregression revealed that possible origination of heterogeneity was baseline IOP before bleb needling, revealing a trend that higher baseline IOP correlated with a greater IOP reduction results $(p<0.001)$. For safety profile, conjunctival haemorrhage $(5.7 \%, 95 \% \mathrm{CI}[2.5 \%, 10.1 \%])$, hyphema $(5.5 \%, 95 \% \mathrm{CI}$ $[3.0 \%, 8.7 \%])$, and bleb leakage $(5.0 \%, 95 \%$ CI $[3.2 \%, 7.3 \%])$ had the highest estimate of incidence. An increasing number of needling was the main risk factor for needling failure. Conclusion. Bleb needling with antimetabolites could be considered an effective and safe procedure after trabeculectomy failure. After the process, patients will gain IOP control and reduce antiglaucomatous medications for at least six months with 5-Fu or MMC. Meanwhile, the overall estimates for complications were relatively low in the whole process.
\end{abstract}

\section{Introduction}

Trabeculectomy is a traditional filtering surgery designed to control intraocular pressure (IOP) with long-term efficacy [1]. With fibrosis and scar formation under the conjunctival and episcleral interface of the filtering bleb, however, IOP is likely to lose control at different follow-up points after the surgery [2]. Bleb needling was designed to rebuild the failing blebs and resolve the unfunctional drainage channel with relatively small injuries. In a setting of operating room or slit-lamp at the clinics, a needle was incised into the subconjunctival space to dissect the adhesion by sweeping motions. Further incision into the anterior chamber may be applied to reopen the scleral flap. Typically, bleb needling with subconjunctival injection of antimetabolites had become a widely accepted approach for the failure of trabeculectomy since the introduction of 5-fluorouracil (5-Fu) [3] and mitomycin C (MMC) [4].

Following the previously mentioned principles, different glaucoma specialists may change the details of practices, according to their own experience [5]. Vast differences were shown in the methodology and clinical settings for the studies of 
bleb needling in the literature. To our knowledge, there were only limited studies about overall estimates for bleb needing with antimetabolites. A previous systemic review about bleb needling without antimetabolites found no conclusive evidence that a single needling outstood conservative antiglaucomatous medication in IOP controlling for encapsulated blebs [6]. As antimetabolites had been used widely, the strict limitation to bleb morphology and lack of updated data had weakened the clinical value of the systemic review. Furthermore, IOP reduction level and success rates after bleb needling varied considerably among studies [2, 4, 7-41]. Comparison and analysis of the possible factors and influence of these between-study variances can strengthen our understanding of the appropriate clinical setting for bleb needling. Therefore, our meta-analysis aimed to shed light on the bleb needling with antimetabolite application after trabeculectomy failure to evaluate the overall IOP reduction effects and complications. To be specific, subscales of antiglaucomatous medications usages, success rates under different definitions, risk factors for failure, and bleb characteristics were simultaneously assessed to provide a more comprehensive view of the safety and efficacy profile of bleb needling.

\section{Methods}

2.1. Search Strategy. The meta-analysis was conducted following the PRISMA guidelines [42]. We performed a systematic electronic search of PubMed, Embase, Cochrane Library, and ClinicalTrials.gov. for articles published until February 2020. The keywords for our search included glaucoma, filtering surgery, trabeculectomy, bleb needling, needling revision, failed bleb, and encapsulated bleb. Relevant publications were examined for references until no further studies were found.

\subsection{Selection Criteria}

\subsubsection{Inclusion Criteria}

(1) Patients diagnosed as having glaucoma, regardless of age, ethnicity, race, or sex; those who underwent bleb needling surgery after trabeculectomy or phacotrabeculectomy failure.

(2) The intervention of bleb needling with subconjunctival injection of antimetabolites surgery due to uncontrolled IOP after trabeculectomy.

(3) The study evaluating our primary outcomes of intraocular pressure before and after bleb needling, or success rate at one year or last visit follow-up.

(4) Studies should be either a randomized-control trial or an observational study. Both comparative and noncomparative studies were included.

\subsubsection{Exclusion Criteria}

(1) Reviews, case reports, non-English studies, published abstracts without available full texts, or reports from meetings
(2) The previous intervention of any drainage implantation, nonpenetrating filter surgery

(3) Bleb needling was not the primary intervention; extra approaches other than bleb needling and subconjunctival injection of medication, or simply an injection of medication without bleb needling will be excluded

(4) Studies about the usage of medications other than a subconjunctival injection of antimetabolites; unclear data of usage were excluded

(5) Studies population from the same trial

(6) Follow-up $<6$ months or unknown

(7) $<10$ people in a group

\subsection{Outcome Measures}

2.3.1. Primary Outcomes. The primary outcomes were mean intraocular pressure (IOP) measured in $\mathrm{mmHg}$ and success rate at the last visit of the follow-up.

2.3.2. Secondary Outcomes. The secondary outcomes were as follows:

(1) Mean IOP and success rate at different time points

(2) The number of antiglaucoma medications

(3) The proportion of participants presenting with a specific adverse event because of bleb needling

(4) Bleb morphology changes

(5) Risk factors for bleb needling failure

2.4. Data Extraction. Two reviewers (XC and $\mathrm{YH}$ ) independently read each title and abstract to exclude irrelevant studies and then reviewed each full text to ascertain its eligibility and to extract data. Any disagreements were resolved by other authors (LS and CZ) and a consensus was reached among all the reviewers. Authors were contacted, if necessary, to acquire any missing information and for unpublished studies.

We extracted data representing the efficacy and safety of bleb needling, including primary outcomes and secondary outcomes. Study characteristics and patients' baseline characteristics were extracted, as well. Different arms for comparative study for MMC and 5-Fu were distributed to different subgroups.

2.5. Risk of Bias Assessment. The risk of bias of studies was evaluated using the checklist recommended by the Agency for Healthcare Research and Quality (AHRQ) [43]. Based on the reviewers' judgment, for each domain (including selection bias, performance bias, detection bias, attrition bias, and reporting bias) on the checklist, every article was rated as having a "low," "high," or "unclear" risk of bias in each domain. Data organization was done using RevMan version 5.3 (Cochrane Community, London, UK). 
2.6. Data Synthesis and Analytical Methods. Data were summarized, and the meta-analysis was performed using Stata version 14.0 (StataCorp, College Station, TX). The IOP and the number of glaucoma medications before and after the bleb needling were compared with a weighted mean difference (WMD). Estimates of complication and success rates were aggregated with double arcsine transmission during our meta-analysis. All the indices were analyzed in a random-effects model, and data were presented as mean with $95 \%$ confidence interval (CI). Besides, we described the heterogeneity by calculating $I^{2}$ values among the studies, which ranged from $0 \%$ to $100 \%$ (with $25 \%, 50 \%$, and $75 \%$ representing low, moderate, and high heterogeneity, resp.). Subgroup analyses were conducted according to antimetabolites, study design, countries or regions, bleb morphology, surgery condition, and acceptance for repeating needling. Metaregression with restricted maximum likelihood (REML) method and Hartung-Knapp method were applied to explore the possible source of heterogeneity. When median with range and size were available in the original articles, data were transformed into mean with standard deviation values as described by Hozo et al. [44]. To detect publication biases, we created funnel plots where asymmetry indicated publication bias. Egger's statistical tests of funnel plot asymmetry were also applied. Sensitivity analysis was performed to evaluate the effect of a single trial on the overall pooled estimate by omitting one trial in each turn. $p<0.05$ was considered statistically significant. Results of our meta-analysis were partially converted to graphs created using Prism 7.0 (GraphPad Software, La Jolla, CA).

\section{Results}

3.1. Search Results. A total of 2126 studies were identified based on the search strategy. After duplication removal, we reviewed the titles and abstracts of 1475 articles and excluded 1377 studies with reasons. The full-text screening was performed for the remaining studies, and finally, 37 full-text articles met the eligibility criteria for meta-analysis. Figure 1 demonstrates the flow diagram of the literature search process.

3.2. Characteristics of Included Studies. The total number of eyes included was 2182 dating from 1993 to 2019. Specifically, 1333 eyes had undergone bleb needling with 5$\mathrm{Fu}$, and 849 eyes, with MMC. Of 37 studies included, there were 25 noncomparative case series and 29 retrospective studies. Twenty-three studies used 5-Fu during bleb needling, while 17 used MMC. Three studies directly compared the efficacy of these antimetabolites $[19,26,29]$. The weighted mean age for all the cases was 63.3 years. The average duration of follow-up after bleb needling ranged from 6 to 60 months. Table 1 summarizes the characteristics of the selected studies. Detailed technique of needling in each article was presented in Supplementary Table 1. Specifically, most of the included studies presented the data of former antimetabolites application of trabeculectomy.
3.3. Bias Assessment. We evaluated the risk of bias of all included articles. Figure 2 displays a summary of bias in each domain with a graph presented in Supplementary Figure 1. The potential existence of publication bias was assessed across the studies by funnel plots in the analysis of IOP and medication changes after bleb needling. No significant asymmetry was found in the test $(p=0.252,0.114,0.150,0.063$ and 0.715 ; for IOP reduction at six months, one year, two years, and last visit, antiglaucomatous medication reduction at last visit, resp.) (Supplementary Figures 2-6).

3.4. IOP Control. IOP reduction levels at six months, one year, two years, and last visit after bleb needling were analyzed. Data at one year were regarded as last-visit data for the studies with loss of $>50 \%$ cases at the last visit [12, 15-17]. Combined analysis with a random-effect model showed a significant reduction in IOP at a different timeline $(p<0.001)$. It decreased by a mean of $8.66 \mathrm{mmHg}(95 \% \mathrm{CI}$ [7.46, 9.85]; $I^{2}=78.4 \% ; 12$ studies) at six months, $8.69 \mathrm{mmHg}\left(95 \% \mathrm{CI}[7.25,10.12] ; I^{2}=85.7 \% ; 13\right.$ studies $)$ at one year, $8.73 \mathrm{mmHg}$ (95\% CI [7.21, 10.25]; $I^{2}=85.1 \%$; 11 studies) at two years (Supplementary Figures 7-9, Figure 3), and $9.74 \mathrm{mmHg}\left(95 \% \mathrm{CI}[8.85,10.63] ; I^{2}=80.9 \% ; 30\right.$ studies $)$ at the last visit. Subgroup analysis was applied to explore sources of heterogeneity among the studies. Typically, bleb needling brought about $9.72 \mathrm{mmHg}(95 \%$ CI [8.41, 11.03]; $\left.I^{2}=81.6 \%\right)$ reduction with MMC injection at the last visit and $9.75 \mathrm{mmHg}\left(95 \% \mathrm{CI}[8.56,10.94] ; I^{2}=79.9 \%\right)$ with 5 -Fu injection. With $95 \% \mathrm{CI}$ from baseline to the last visit, WMD was presented as a forest plot (Figure 4). Of note, most of the strata in the subgroup showed high heterogeneity (Supplementary Table 2). Sensitivity analysis revealed that the estimate of WMD was stable (Supplementary Figure 11). Further metaregression revealed that IOP before bleb needling contributed significantly to the heterogeneity of WMD of IOP reduction at last visit $(p<0.001)$, revealing a trend that higher baseline IOP correlated with a greater IOP reduction results (Figure 5, Supplementary Table 3). To be specific, it explained $64.9 \%$ of the between-study variance.

3.5. Estimate for the Success Rate. Criteria of success varied among studies. In reviewing the studies, the most accepted definition of complete success was IOP $<21 \mathrm{mmHg}$ or reduction of $20 \%$ from baseline without any antiglaucomatous medication $[2,7,9,11,13,15,19-22,30,31,36,38]$, while qualified success referred to the same IOP level with topical antiglaucomatous medication $[7,9,11,12,15,19-22,30,31,36,38]$. Those with criteria stricter than the previously mentioned definition were considered eligible for the pooled analysis of success rate. In a random-effect model, the overall estimate of complete success rate for bleb needling at the last visit was 45.9\% (95\% CI [39.0\%, 53.0\%]; $I^{2}=84.1 \%$; 22 studies), while qualified success rate was $70.4 \%$ (95\% CI [63.5\%, 77.0\%]; $I^{2}=88.1 \% ; 26$ studies). Specifically, MMC injection subgroup reached $48.6 \%$ (95\% CI [35.0\%, $62.2 \%]$ ) in complete success rate and $70.2 \%$ (95\% CI [60.0\%, $79.7 \%]$ ) in qualified success rate, while 5-Fu subgroup was $44.3 \%$ (95\% CI $[36.3 \%, 52.4 \%])$ and $70.7 \%(95 \%$ CI $[60.9 \%, 79.6 \%])$. 


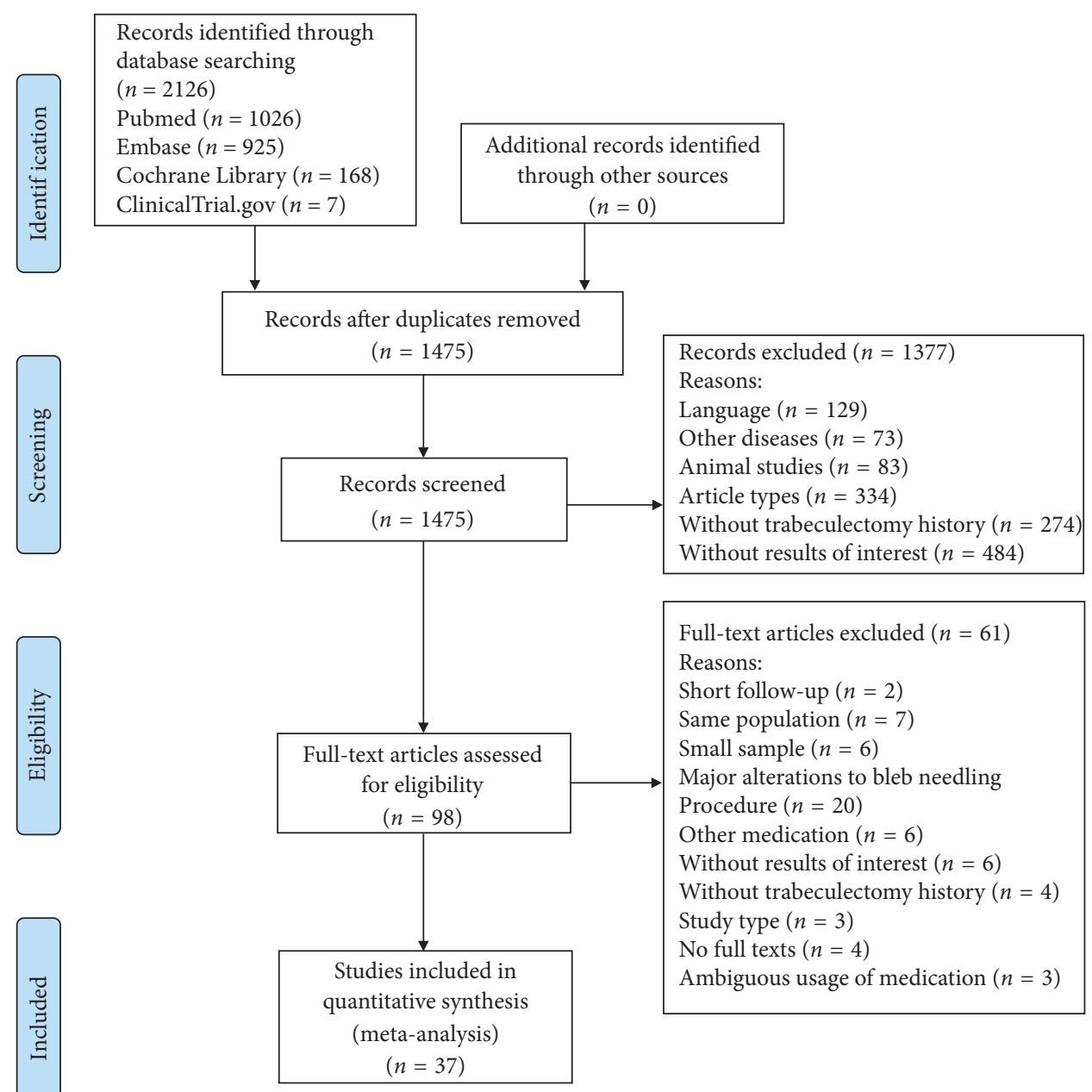

FIgURE 1: Flow diagram of literature search and included studies for meta-analysis.

3.6. Antiglaucomatous Medication Reduction. Twenty studies had a record of antiglaucomatous medication usage before and after bleb needling. The postoperative number of medications was significantly fewer than the preoperative number of medications by 0.80 (95\% CI [0.52, 1.09]; $p<0.001$; Supplementary Figure 10).

3.7. Bleb Morphology. Needling will alter the morphology of failed bleb, though data are presented in limited studies $[13,17,29,35]$. Flat blebs $[13,17,35]$ and scared blebs [29], as the primary form of bleb characteristic in the studies, significantly shifted into diffuse blebs after needling $[13,35]$. Four studies $[11,23,30,40]$ had focused on encapsulated bleb while one study [25] shed light on flat filtering bleb. However, no quantitative descriptions of morphology changes were presented in these studies.

As far as the relationship between bleb morphology and surgical outcomes was concerned, only seven studies were involved $[2,7,8,13,17,20,35]$. Based on different descriptions for bleb characteristics, the conclusion varied among studies. Lee et al. [20] claimed that greater central bleb extension and more elevated height were associated with a higher chance of success. Than et al. [13] showed that injected bleb appearance before needling was a significant indicator of success. Rotchford and King [2] considered bleb morphology a modifier rather than a risk factor for surgical outcomes. They displayed that when the bleb was injected or microcystic, bleb elevation at the time of needling correlated with longer survival time. However, IOP reduction had no significant difference in comparison of flat blebs and other types before needling $[7,8,17]$. While under a definition of postneedling IOP $<22 \mathrm{mmHg}$, or $30 \%$ reduction of IOP, the variation of success rate between bleb types did not reach statistical significance [35]. The predictive value for bleb morphology remained controversial.

3.8. Complications. A total of 29 studies, including 1661 cases, reported the occurrence of complications. We ran the meta-analysis based on these studies. An estimate of the incidence rate of complication throughout the whole course of study following bleb needling is presented in Table 2 . Within all the complications, conjunctival haemorrhage (5.7\%, 95\% CI [2.5\%, 10.1\%]), hyphema (5.5\%, 95\% CI [3.0\%, 8.7\%]), and bleb leakage (5.0\%, 95\% CI [3.2\%, 7.3\%]) were the most commonly reported and had the highest estimate of incidence. Most of them can be resolved by 
TABLE 1: Baseline characteristics of included studies.

\begin{tabular}{|c|c|c|c|c|c|c|c|c|}
\hline Author, year & Design & $\begin{array}{c}\text { Number } \\
\text { of eyes }\end{array}$ & $\begin{array}{l}\text { Mean age } \\
(\mathrm{SD}) \\
\text { (years) }\end{array}$ & $\begin{array}{l}\text { Mean } \\
\text { follow-up } \\
\text { (SD) } \\
\text { (months) }\end{array}$ & $\begin{array}{l}\text { Diagnosis for study } \\
\text { eyes (numbers (\%)) }\end{array}$ & $\begin{array}{l}\text { Phakic status for } \\
\text { study eyes } \\
\text { (numbers }(\%))\end{array}$ & Medication & $\begin{array}{l}\text { Country/ } \\
\text { region }\end{array}$ \\
\hline $\begin{array}{l}\text { Rabiolo et al., } \\
2019 \text { [10] }\end{array}$ & $\begin{array}{l}\text { Noncomparative, } \\
\text { retrospective }\end{array}$ & 157 & $\begin{array}{c}65.8 \\
(13.9)\end{array}$ & $25(30.37)$ & $\begin{array}{c}\text { POAG: } 85(54.1) \\
\text { PACG: } 11(7.0) \\
\text { Secondary } \\
\text { glaucoma: } 61(38.9)\end{array}$ & $\begin{array}{c}\text { Pseudo: } 140 \\
\text { (89.2); phakic: } 16 \\
(10.2) \text {; aphakic: } 1 \\
(0.6)\end{array}$ & $5-\mathrm{Fu}$ & Italy \\
\hline $\begin{array}{l}\text { Okka et al., } \\
2019 \text { [11] }\end{array}$ & $\begin{array}{l}\text { Noncomparative, } \\
\text { retrospective }\end{array}$ & 16 & $46(18.2)$ & $\begin{array}{l}12.22 \\
(6.08)\end{array}$ & $\begin{array}{l}\text { POAG: } 9(56.3) \\
\text { Secondary } \\
\text { glaucoma: } 7(44) \\
\text { POAG: } 21(60.0)\end{array}$ & N/A & $5-\mathrm{Fu}$ & Turkey \\
\hline $\begin{array}{l}\text { Kim et al., } \\
2019 \text { [12] }\end{array}$ & $\begin{array}{l}\text { Comparative, } \\
\text { retrospective }\end{array}$ & 35 & $\begin{array}{c}58.5 \\
(13.1)\end{array}$ & $>6(\mathrm{~N} / \mathrm{A})$ & $\begin{array}{c}\text { PACG: } 3(8.6) \\
\text { Secondary } \\
\text { glaucoma: } 11(31.4)\end{array}$ & N/A & $5-\mathrm{Fu}$ & $\begin{array}{l}\text { South } \\
\text { Korea }\end{array}$ \\
\hline $\begin{array}{l}\text { Than et al., } \\
2018 \text { [13] }\end{array}$ & $\begin{array}{l}\text { Noncomparative, } \\
\text { retrospective }\end{array}$ & 96 & $67(15)$ & $37.08(21)$ & $\begin{array}{c}\text { POAG: } 70(72.9) \\
\text { PACG: } 6(6.25) \\
\text { Secondary } \\
\text { glaucoma: } 18 \\
(18.75) \\
\text { N/A: } 2(2.1)\end{array}$ & $\begin{array}{c}\text { Pseudo: } 24(25) \text {; } \\
\text { phakic: } 72(75)\end{array}$ & $5-\mathrm{Fu}$ & UK \\
\hline $\begin{array}{l}\text { Pathak-Ray } \\
\text { and } \\
\text { Choudhari, } \\
2018 \text { [14] }\end{array}$ & $\begin{array}{c}\text { Noncomparative, } \\
\text { prospective }\end{array}$ & 39 & $\begin{array}{c}59 \\
(14.25)\end{array}$ & $20(11)$ & $\begin{array}{c}\text { POAG: } 9(23.1) \\
\text { PACG: } 21(53.8) \\
\text { Secondary } \\
\text { glaucoma: } 9(23.1)\end{array}$ & $\begin{array}{c}\text { Pseudo: } 26 \\
\text { (66.7); phakic: } 13 \\
(33.3)\end{array}$ & MMC & India \\
\hline $\begin{array}{l}\text { Lin et al., } 2018 \\
{[15]}\end{array}$ & $\begin{array}{l}\text { Noncomparative, } \\
\text { retrospective }\end{array}$ & 44 & $\begin{array}{c}73.7(\mathrm{~N} / \\
\mathrm{A})\end{array}$ & $58.7(\mathrm{~N} / \mathrm{A})$ & $\begin{array}{c}\text { POAG: } 29(65.9) \\
\text { PACG: } 7(15.9) \\
\text { Secondary } \\
\text { glaucoma: } 8(18.2)\end{array}$ & $\begin{array}{c}\text { Pseudo: } 28 \\
\text { (63.6); phakic: } 16 \\
(36.4)\end{array}$ & MMC & UK \\
\hline $\begin{array}{l}\text { Zheng et al., } \\
2016 \text { [16] }\end{array}$ & $\begin{array}{l}\text { Noncomparative, } \\
\text { retrospective }\end{array}$ & 33 & $\begin{array}{c}68.67 \\
(10.38)\end{array}$ & $\begin{array}{c}21.72 \\
(18.72)\end{array}$ & $\begin{array}{c}\text { POAG: } 21(67.7) \\
\text { PACG: } 2(6.5) \\
\text { Secondary } \\
\text { glaucoma: } 8(25.8)^{\#}\end{array}$ & N/A & $5-\mathrm{Fu}$ & Australia \\
\hline $\begin{array}{l}\text { Tulidowicz- } \\
\text { Bielak et al., } \\
2016 \text { [17] }\end{array}$ & $\begin{array}{l}\text { Noncomparative, } \\
\text { retrospective }\end{array}$ & 121 & $\begin{array}{c}67.38 \\
(13.76)\end{array}$ & $27.6(\mathrm{~N} / \mathrm{A})$ & POAG: 121 (100) & $\begin{array}{c}\text { Single needling: } \\
\text { pseudo: } 62(51.2) \text {; } \\
\text { phakic: } 38 \text { (31.4); } \\
\text { multiple } \\
\text { needling: } \\
\text { unknown } 21 \\
(17.4)\end{array}$ & MMC & Poland \\
\hline $\begin{array}{l}\text { Panarelli et al., } \\
2016 \text { [18] }\end{array}$ & $\begin{array}{l}\text { Noncomparative, } \\
\text { retrospective }\end{array}$ & 27 & $\begin{array}{c}69.1 \\
(13.1)\end{array}$ & $54.2(30.4)$ & $\begin{array}{c}\text { POAG: } 18 \text { (66.7) } \\
\text { PACG: } 2(7.4) \\
\text { Secondary } \\
\text { glaucoma: } 7 \text { (25.9) }\end{array}$ & $\begin{array}{c}\text { Pseudo: } 17 \\
\text { (63.0); phakic: } 9 \\
\text { (33.3); aphakic: } 1 \\
\text { (3.7) }\end{array}$ & MMC & USA \\
\hline $\begin{array}{l}\text { Liu et al., } 2016 \\
{[19]}\end{array}$ & RCT & 75 & $\begin{array}{c}48.5 \\
(10.4)\end{array}$ & $12(\mathrm{~N} / \mathrm{A})$ & $\begin{array}{c}\text { MMC: POAG: } 14 \\
\text { (35.0) PACG: } 23 \\
\text { (57.5) } \\
\text { Juvenile glaucoma: } \\
3 \text { (7.5) 5-Fu: } \\
\text { POAG: } 12(34.3) \\
\text { PACG: } 20 \text { (57.1) } \\
\text { Juvenile glaucoma: } \\
1 \text { (2.9) } \\
\text { Secondary } \\
\text { glaucoma: } 2 \text { (5.7) }\end{array}$ & N/A & $\begin{array}{c}\mathrm{MMC} / 5- \\
\mathrm{Fu}\end{array}$ & China \\
\hline $\begin{array}{l}\text { Lee et al., } 2016 \\
{[20]}\end{array}$ & $\begin{array}{l}\text { Comparative, } \\
\text { retrospective }\end{array}$ & 41 & $\begin{array}{c}52.1 \\
(15.8)\end{array}$ & $22.7(9.4)$ & $\begin{array}{c}\text { POAG: } 8(19.5) \\
\text { PACG: } 5(12.2) \\
\text { Secondary } \\
\text { glaucoma: } 28(68.3)\end{array}$ & $\begin{array}{c}\text { Pseudo: } 19 \\
\text { (46.3); phakic: } 21 \\
\text { (51.2); aphakic: } 1 \\
\text { (2.4) }\end{array}$ & $5-\mathrm{Fu}$ & China \\
\hline
\end{tabular}


TABle 1: Continued.

\begin{tabular}{|c|c|c|c|c|c|c|c|c|}
\hline Author, year & Design & $\begin{array}{c}\text { Number } \\
\text { of eyes }\end{array}$ & $\begin{array}{l}\text { Mean age } \\
(\mathrm{SD}) \\
\text { (years) }\end{array}$ & $\begin{array}{l}\text { Mean } \\
\text { follow-up } \\
\text { (SD) } \\
\text { (months) }\end{array}$ & $\begin{array}{l}\text { Diagnosis for study } \\
\text { eyes (numbers (\%)) }\end{array}$ & $\begin{array}{c}\text { Phakic status for } \\
\text { study eyes } \\
\text { (numbers }(\%) \text { ) }\end{array}$ & Medication & $\begin{array}{l}\text { Country/ } \\
\text { region }\end{array}$ \\
\hline $\begin{array}{l}\text { Tsai et al., } 2015 \\
{[21]}\end{array}$ & $\begin{array}{l}\text { Comparative, } \\
\text { retrospective }\end{array}$ & 227 & $\begin{array}{c}66.9 \\
(11.2)\end{array}$ & $>6(\mathrm{~N} / \mathrm{A})$ & $\begin{array}{l}\text { POAG: } 157(69.2) \\
\text { PACG: } 70(30.8)\end{array}$ & N/A & $5-\mathrm{Fu}$ & Singapore \\
\hline $\begin{array}{l}\text { Tatham et al., } \\
2013 \text { [22] }\end{array}$ & $\begin{array}{l}\text { Comparative, } \\
\text { retrospective }\end{array}$ & 34 & $\begin{array}{c}67.88(\mathrm{~N} / \\
\mathrm{A})\end{array}$ & $25.2(1.2)$ & $\begin{array}{c}\text { POAG: } 21(61.8) \\
\text { PACG: } 5(14.7) \\
\text { Secondary } \\
\text { glaucoma: } 8(23.5)\end{array}$ & $\begin{array}{c}\text { Pseudo: } 17 \\
\text { (50.0); phakic: } 17 \\
(50.0)\end{array}$ & $5-\mathrm{Fu}$ & UK \\
\hline $\begin{array}{l}\text { Suzuki and } \\
\text { Susanna-Jr, } \\
2013 \text { [23] }\end{array}$ & RCT & 20 & $\begin{array}{c}57.3 \\
(15.21)\end{array}$ & $12(\mathrm{~N} / \mathrm{A})$ & $\begin{array}{l}\text { POAG: } 17(85.0) \\
\text { Congenital } \\
\text { glaucoma: } 1(5.0) \\
\text { Secondary } \\
\text { glaucoma: } 2(10.0)\end{array}$ & N/A & $5-\mathrm{Fu}$ & Brazil \\
\hline $\begin{array}{l}\text { Dalvi et al., } \\
2012 \text { [24] }\end{array}$ & $\begin{array}{l}\text { Comparative, } \\
\text { retrospective }\end{array}$ & 40 & $\begin{array}{l}67.83 \\
(12.02)\end{array}$ & $60(\mathrm{~N} / \mathrm{A})$ & $\begin{array}{c}\text { POAG: } 28(70.0) \\
\text { PACG: } 6(15.0) \\
\text { Secondary } \\
\text { glaucoma: } 6(15.0)\end{array}$ & $\begin{array}{c}\text { Pseudo: } 20 \\
\text { (50.0); phakic: } 20 \\
(50.0)\end{array}$ & $5-\mathrm{Fu}$ & Canada \\
\hline $\begin{array}{l}\text { Amini et al., } \\
2012 \text { [7] }\end{array}$ & $\begin{array}{l}\text { Noncomparative, } \\
\text { retrospective }\end{array}$ & 27 & $\begin{array}{c}56.5 \\
(16.2)\end{array}$ & $\begin{array}{c}20.31 \\
(15.63)\end{array}$ & $\begin{array}{c}\text { POAG: } 7 \text { (26.0) } \\
\text { PACG: } 4 \text { (14.8) } \\
\text { Juvenile glaucoma: } \\
4 \text { (14.8) } \\
\text { Secondary } \\
\text { glaucoma: } 11 \text { (40.7) } \\
\text { Developmental } \\
\text { glaucoma: } 1(3.7)\end{array}$ & N/A & MMC & Iran \\
\hline $\begin{array}{l}\text { Maestrini } \\
\text { et al., } 2011 \text { [25] }\end{array}$ & $\begin{array}{c}\text { Noncomparative, } \\
\text { prospective }\end{array}$ & 125 & $\begin{array}{c}61.6 \\
(18.8)\end{array}$ & $20.8(12)$ & $\begin{array}{l}\text { POAG: } 97 \text { (77.6) } \\
\text { PACG: } 10(8.0) \\
\text { Congenital } \\
\text { glaucoma: } 6(4.8) \\
\text { Secondary } \\
\text { glaucoma: } 12(9.6)\end{array}$ & $\begin{array}{c}\text { Pseudo: } 53 \\
\text { (42.4); phakic: } 69 \\
\text { (55.2); aphakic: } 3 \\
(2.4)\end{array}$ & MMC & Brazil \\
\hline $\begin{array}{l}\text { Palejwala et al., } \\
2010 \text { [26] }\end{array}$ & $\begin{array}{l}\text { Comparative, } \\
\text { retrospective }\end{array}$ & 107 & $\begin{array}{c}74.35 \\
(11.95)\end{array}$ & $\begin{array}{c}10.55 \\
(11.01)\end{array}$ & $\begin{array}{c}\text { POAG: } 81(75.7) \\
\text { PACG: } 3(2.8) \\
\text { Secondary } \\
\text { glaucoma: } 23(21.5)\end{array}$ & $\begin{array}{c}\text { Pseudo: } 48 \\
\text { (44.9); phakic: } 58 \\
(54.2) \text {; aphakic: } 1 \\
(0.9)\end{array}$ & $\begin{array}{c}\mathrm{MMC} / 5- \\
\mathrm{Fu}\end{array}$ & USA \\
\hline $\begin{array}{l}\text { Elsayed and } \\
\text { El-Raggal, } \\
2010 \text { [27] }\end{array}$ & $\begin{array}{l}\text { Noncomparative, } \\
\text { retrospective }\end{array}$ & 30 & $7.3(3.4)$ & $9.23(5.25)$ & $\begin{array}{c}\text { Congenital } \\
\text { glaucoma: } 30(100)\end{array}$ & N/A & MMC & Egypt \\
\hline $\begin{array}{l}\text { Kapasi and } \\
\text { Birt, } 2009 \text { [28] }\end{array}$ & $\begin{array}{l}\text { Noncomparative, } \\
\text { retrospective }\end{array}$ & 37 & $\begin{array}{c}71.2 \\
(12.6)\end{array}$ & $24(\mathrm{~N} / \mathrm{A})$ & $\mathrm{N} / \mathrm{A}$ & N/A & $5-\mathrm{Fu}$ & Canada \\
\hline $\begin{array}{l}\text { Anand and } \\
\text { Khan, } 2009 \\
{[29]}\end{array}$ & $\begin{array}{c}\text { Noncomparative, } \\
\text { prospective }\end{array}$ & 33 & $\begin{array}{l}45.67 \\
(22.41)\end{array}$ & $9.24(5.27)$ & $\begin{array}{c}\text { 5-Fu: POAG: } 45 \\
(88.2) \\
\text { PACG: } 5(9.8) \\
\text { Secondary } \\
\text { glaucoma: } 1 \text { (2.0) } \\
\text { MMC: POAG: } 37 \\
\text { (841) } \\
\text { PACG: } 4 \text { (9.0) } \\
\text { Secondary } \\
\text { glaucoma: } 3(6.9)^{\#} \\
\text { POAG: } 9 \text { (27.3) } \\
\text { PACG: } 4 \text { (12.1) } \\
\text { Congenital } \\
\text { glaucoma: } 6(18.2) \\
\text { Juvenile glaucoma: } \\
4 \text { (12.1) } \\
\text { Developmental } \\
\text { glaucoma: } 1(2.7) \\
\text { Secondary } \\
\text { glaucoma: } 9 \text { (27.3) }\end{array}$ & $\begin{array}{l}\text { MMC group: } \\
\text { pseudo: } 28 \\
\text { (62.2); phakic: } 17 \\
\text { (37.8); } 5 \text {-Fu } \\
\text { group: pseudo: } \\
30 \text { (56.6); phakic: } \\
23 \text { (43.4) }\end{array}$ & $\begin{array}{c}\mathrm{MMC} / 5- \\
\mathrm{Fu}\end{array}$ & Iran \\
\hline
\end{tabular}


TABle 1: Continued.

\begin{tabular}{|c|c|c|c|c|c|c|c|c|}
\hline Author, year & Design & $\begin{array}{c}\text { Number } \\
\text { of eyes }\end{array}$ & $\begin{array}{l}\text { Mean age } \\
(\mathrm{SD}) \\
\text { (years) }\end{array}$ & $\begin{array}{l}\text { Mean } \\
\text { follow-up } \\
\text { (SD) } \\
\text { (months) }\end{array}$ & $\begin{array}{l}\text { Diagnosis for study } \\
\text { eyes (numbers (\%)) }\end{array}$ & $\begin{array}{c}\text { Phakic status for } \\
\text { study eyes } \\
\text { (numbers }(\%))\end{array}$ & Medication & $\begin{array}{l}\text { Country/ } \\
\text { region }\end{array}$ \\
\hline $\begin{array}{l}\text { Rotchford and } \\
\text { King, 2008 [2] }\end{array}$ & $\begin{array}{c}\text { Noncomparative, } \\
\text { prospective }\end{array}$ & 81 & 70 (N/A) & $\begin{array}{c}40.27(\mathrm{~N} / \\
\mathrm{A})\end{array}$ & $\begin{array}{c}\text { POAG: } 56(69.1) \\
\text { PACG: } 8(9.9) \\
\text { secondary } \\
\text { glaucoma: } 17(21.0)\end{array}$ & $\begin{array}{c}\text { Pseudo: } 27 \\
\text { (33.3); phakic: } 54 \\
(66.7)\end{array}$ & $5-\mathrm{Fu}$ & UK \\
\hline $\begin{array}{l}\text { Gutierrez- } \\
\text { Ortiz et al., } \\
2006 \text { [31] }\end{array}$ & $\begin{array}{c}\text { Noncomparative, } \\
\text { prospective }\end{array}$ & 34 & $65.9(8.3)$ & $14.2(9.8)$ & $\begin{array}{c}\text { POAG: } 23 \text { (67.6) } \\
\text { PACG: } 5 \text { (14.7) } \\
\text { Juvenile glaucoma: } \\
1 \text { (2.9) } \\
\text { Secondary } \\
\text { glaucoma: } 5 \text { (14.7) }\end{array}$ & N/A & MMC & Spain \\
\hline $\begin{array}{l}\text { Shetty et al., } \\
2005 \text { [32] }\end{array}$ & $\begin{array}{l}\text { Noncomparative, } \\
\text { retrospective }\end{array}$ & 44 & $\begin{array}{c}72.9 \\
(10.4)\end{array}$ & $>12(\mathrm{NA})$ & $\begin{array}{c}\text { POAG: } 35 \text { (79.5) } \\
\text { PACG: } 4(9.1) \\
\text { Secondary } \\
\text { glaucoma: } 5(11.4)\end{array}$ & $\begin{array}{c}\text { Pseudo: } 31 \\
\text { (70.5); phakic: } 12 \\
\text { (27.3); aphakic: } 1 \\
\text { (2.3) }\end{array}$ & MMC & USA \\
\hline $\begin{array}{l}\text { Jacobs et al., } \\
2005[33]\end{array}$ & $\begin{array}{l}\text { Noncomparative, } \\
\text { retrospective }\end{array}$ & 28 & $61(\mathrm{~N} / \mathrm{A})$ & $14(\mathrm{~N} / \mathrm{A})$ & $\mathrm{N} / \mathrm{A}$ & N/A & $5-\mathrm{Fu}$ & Belgium \\
\hline $\begin{array}{l}\text { Paris et al., } \\
2004 \text { [34] }\end{array}$ & $\begin{array}{l}\text { Noncomparative, } \\
\text { retrospective }\end{array}$ & 36 & N/A & $6(\mathrm{~N} / \mathrm{A})$ & POAG: 36 (100) & N/A & $5-\mathrm{Fu}$ & USA \\
\hline $\begin{array}{l}\text { Broadway } \\
\text { et al., } 2004 \text { [35] }\end{array}$ & $\begin{array}{l}\text { Noncomparative, } \\
\text { retrospective }\end{array}$ & 101 & $69(11)$ & 20.2 (NA) & $\begin{array}{c}\text { POAG: } 71(70.2) \\
\text { PACG: } 5(5.0) \\
\text { Secondary } \\
\text { glaucoma: } 25(24.8) \\
\text { POAG: } 26(63.4)\end{array}$ & $\begin{array}{l}\text { Pseudo: } 16(15.8) \text {; } \\
\text { phakic: } 12(79.2) \text {; } \\
\text { aphakic: } 5(5.0)\end{array}$ & $5-\mathrm{Fu}$ & UK \\
\hline $\begin{array}{l}\text { Ben-Simon } \\
\text { and Glovinsky, } \\
2003 \text { [36] }\end{array}$ & $\begin{array}{l}\text { Noncomparative, } \\
\text { retrospective }\end{array}$ & 41 & $65(17)$ & $6(\mathrm{~N} / \mathrm{A})$ & $\begin{array}{c}\text { PACG: } 2(4.9) \\
\text { Congenital } \\
\text { glaucoma: } 2(4.9) \\
\text { Secondary } \\
\text { glaucoma: } 11(26.8)\end{array}$ & $\begin{array}{c}\text { Pseudo: } 22 \\
\text { (53.7); phakic: } 13 \\
\text { (31.7); aphakic: } 6 \\
\text { (14.6) }\end{array}$ & MMC & Israel \\
\hline $\begin{array}{l}\text { Hawkins et al., } \\
2002 \text { [37] }\end{array}$ & $\begin{array}{l}\text { Noncomparative, } \\
\text { retrospective }\end{array}$ & 49 & $\begin{array}{c}70.14 \\
(13.74)\end{array}$ & $19.9(16.3)$ & $\begin{array}{c}\text { POAG: } 25(58.1) \\
\text { PACG: } 8 \text { (18.6) } \\
\text { Secondary } \\
\text { glaucoma: } 10(23.3) \\
\#\end{array}$ & $\begin{array}{c}\text { Pseudo/aphakic: } \\
32 \text { (74.4); phakic: } \\
11(25.6)\end{array}$ & $5-\mathrm{Fu}$ & USA \\
\hline $\begin{array}{l}\text { Chang and } \\
\text { Hou, } 2002 \text { [38] }\end{array}$ & $\begin{array}{l}\text { Noncomparative, } \\
\text { retrospective }\end{array}$ & 25 & $\begin{array}{c}53.0 \\
(13.2)\end{array}$ & $8.32(6.61)$ & $\begin{array}{c}\text { POAG: } 4(16.0) \\
\text { PACG: } 4(16.0) \\
\text { Secondary } \\
\text { glaucoma: } 17(68.0)\end{array}$ & $\begin{array}{c}\text { Pseudo: } 12 \\
\text { (48.0); phakic: } 6 \\
(24.0) \text {; aphakic: } 7 \\
(28.0)\end{array}$ & $5-\mathrm{Fu}$ & China \\
\hline $\begin{array}{l}\text { Shin et al., } \\
2001 \text { [39] }\end{array}$ & $\begin{array}{l}\text { Noncomparative, } \\
\text { retrospective }\end{array}$ & 64 & $72.1(9.5)$ & $44.2(21.2)$ & $\begin{array}{c}\text { POAG: } 61 \text { (95.3) } \\
\text { PACG: } 2 \text { (3.1) } \\
\text { Secondary } \\
\text { glaucoma: } 1 \text { (1.6) }\end{array}$ & $\begin{array}{c}\text { Pseudo: } 10(15.6) \text {; } \\
\text { phakic: } 51 \text { (79.7); } \\
\text { aphakic: } 3(4.7)\end{array}$ & $5-\mathrm{Fu}$ & USA \\
\hline $\begin{array}{l}\text { Kapetansky } \\
\text { and } \\
\text { Kapetansky, } \\
1999[8]\end{array}$ & $\begin{array}{l}\text { Comparative, } \\
\text { retrospective }\end{array}$ & 30 & N/A & $>6(\mathrm{~N} / \mathrm{A})$ & N/A & N/A & MMC & USA \\
\hline $\begin{array}{l}\text { Allen et al., } \\
1998 \text { [40] }\end{array}$ & $\begin{array}{l}\text { Noncomparative, } \\
\text { retrospective }\end{array}$ & 32 & $\begin{array}{c}63.9 \\
(10.8)\end{array}$ & $10.7(2.9)$ & $\begin{array}{c}\text { POAG: } 20(62.5) \\
\text { PACG: } 5(15.6) \\
\text { Secondary } \\
\text { glaucoma: } 7 \text { (21.9) } \\
\text { POAG: } 32(51.6)\end{array}$ & $\begin{array}{c}\text { Pseudo: } 6(18.8) \text {; } \\
\text { phakic: } 24(75.0) \text {; } \\
\text { aphakic: } 2(6.2)\end{array}$ & $5-\mathrm{Fu}$ & UK \\
\hline $\begin{array}{l}\text { Mardelli et al., } \\
1996 \text { [4] }\end{array}$ & $\begin{array}{l}\text { Noncomparative, } \\
\text { retrospective }\end{array}$ & 62 & $72(15)$ & $9.9(3.7)$ & $\begin{array}{c}\text { PACG: } 9 \text { (14.5) } \\
\text { Congenital } \\
\text { glaucoma: } 4(6.5) \\
\text { Secondary } \\
\text { glaucoma: } 17(27.4)\end{array}$ & $\begin{array}{c}\text { Pseudo: } 42 \\
\text { (67.8); phakic: } 17 \\
\text { (27.4); aphakic: } 3 \\
(4.8)\end{array}$ & MMC & USA \\
\hline $\begin{array}{l}\text { Greenfield } \\
\text { et al., } 1996 \text { [41] }\end{array}$ & $\begin{array}{l}\text { Comparative, } \\
\text { retrospective }\end{array}$ & 63 & $\begin{array}{c}72.3 \\
(11.2)\end{array}$ & $13.1(8.1)$ & $\begin{array}{c}\text { POAG: } 32(50.8) \\
\text { PACG: } 9(14.3) \\
\text { Secondary } \\
\text { glaucoma: } 22(34.9)\end{array}$ & $\begin{array}{c}\text { Pseudo: } 27 \\
\text { (42.9); phakic: } 36 \\
\text { (57.1) }\end{array}$ & MMC & USA \\
\hline
\end{tabular}


TABle 1: Continued.

\begin{tabular}{|c|c|c|c|c|c|c|c|c|}
\hline Author, year & Design & $\begin{array}{l}\text { Number } \\
\text { of eyes }\end{array}$ & $\begin{array}{l}\text { Mean age } \\
(\mathrm{SD}) \\
\text { (years) }\end{array}$ & $\begin{array}{l}\text { Mean } \\
\text { follow-up } \\
\text { (SD) } \\
\text { (months) }\end{array}$ & $\begin{array}{l}\text { Diagnosis for study } \\
\text { eyes (numbers (\%)) }\end{array}$ & $\begin{array}{l}\text { Phakic status for } \\
\text { study eyes } \\
\text { (numbers }(\%))\end{array}$ & Medication & $\begin{array}{l}\text { Country/ } \\
\text { region }\end{array}$ \\
\hline $\begin{array}{l}\text { Shin et al., } \\
1993 \text { [9] }\end{array}$ & $\begin{array}{l}\text { Comparative, } \\
\text { retrospective }\end{array}$ & 30 & $\begin{array}{c}63.5 \\
(14.5)\end{array}$ & $15.5(5.2)$ & $\begin{array}{c}\text { POAG: } 22(73.3) \\
\text { Secondary } \\
\text { glaucoma: } 8(26.7)\end{array}$ & $\begin{array}{c}\text { Pseudo: } 17 \\
\text { (56.7); phakic: } 10 \\
\text { (33.3); aphakic: } 3 \\
\text { (10.0) }\end{array}$ & $5-\mathrm{Fu}$ & USA \\
\hline
\end{tabular}

*MMC: mitomycin C; PACG: primary angle-closure glaucoma; POAG: primary open-angle glaucoma; RCT: randomized-control trial; SD: standard deviation; 5-Fu: 5-fluorouracil. N/A meant that the data were not presented in the original article. ${ }^{* *}$ Secondary glaucoma included pseudoexfoliated glaucoma, uveitic glaucoma, pigmentary glaucoma, traumatic glaucoma, steroid-induced glaucoma, neovascular glaucoma, iridocorneal endothelial syndrome, and so on. Developmental glaucoma included Axenfeld-Reiger Syndrome, Sturge-Weber Syndrome, and so on. ${ }^{\#}$ Number of patients was displayed.

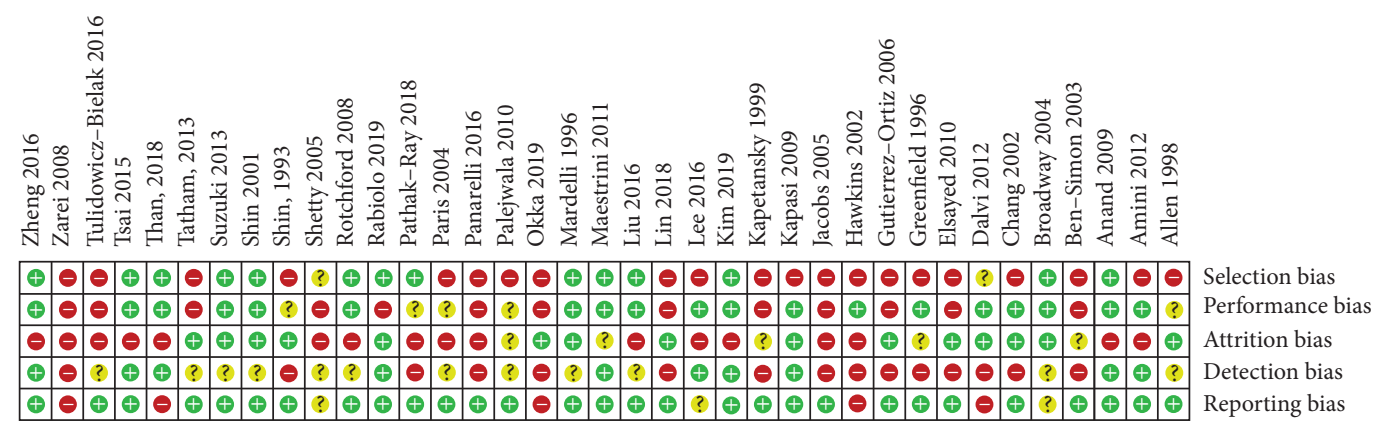

FIGURE 2: Summary of risk of bias assessment for each selected case.
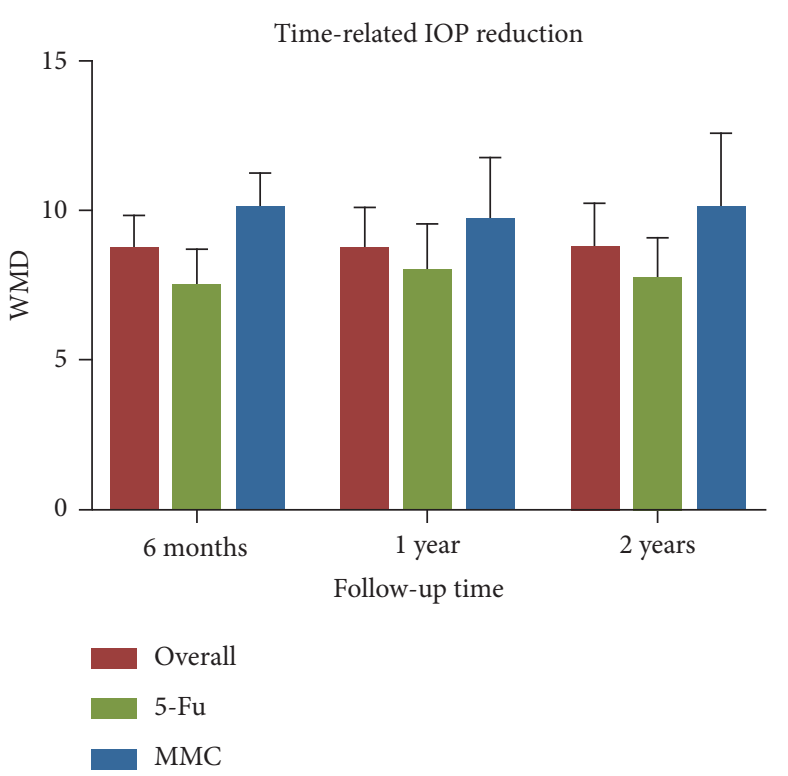

FIGURE 3: Column graph for time-related IOP reduction: weighted mean difference (WMD) of reduction at different timeline showed stable effects for bleb needling with 5-Fu and MMC.

conservative treatment or after observation. According to our estimation, complications that appeared after MMC injection might be slightly more than that of $5-\mathrm{Fu}$ injection, especially in conjunctival haemorrhage (10.8\% vs. $2.7 \%)$. Besides, some rare complications were listed as they were not suitable for aggregation (events/cases): vitreous haemorrhage $(4 \%, 5 / 125)[25]$, cystoid macular oedema $(2.4 \%, 4 /$
266) $[2,10,33]$, bullous keratopathy $(1.7 \%, 5 / 290)$ $[11,17,29,31,39]$, corneal endothelial decompensation $(1.5 \%, 5 / 330)[2,10,14,29]$, suprachoroidal haemorrhage $(1.4 \%, 6 / 422)[2,4,17,26,29,41]$, endophthalmitis $(1.4 \%, 3 /$ $220)[10,41]$, hypotony maculopathy $(1.3 \%, 2 / 157)[10]$, and blebitis $(0.6 \%, 1 / 157)[10]$. The variance of incidence among studies was probably caused by the setting of different trials. Those with a longer duration of follow-up $[15,18,29]$ and larger sample size $[10,17,25,35]$ reported relatively more complications. Typically, Maestrini et al. [25] reported a maximum of 173 cases of postoperative complications in a mean follow-up of 20.8 months for 125 eyes. Cataract was reported in four studies, but its correlation with bleb needling was uncertain $[10,14,25,33]$.

3.9. Possible Risk Factors for Surgical Outcome. Potential risk factors for the failure of bleb needling had been studied among 11 studies, mainly focusing on the number of needling, IOP, and time interval between needling and trabeculectomy [2, 10, 12-14, 20, 21, 29, 35, 37, 39].

Five studies concluded that the total number of needling was an independent risk factor [2, 12-14, 21]. In a long-term study, both median survival time and two-year success rate were significantly reduced for those who underwent more than one needling [2]. One study had calculated that the hazard ratio (HR) was 16.13 (95\% CI, [1.22, 211.71]) [14]. However, given that this particular research was based on data with a wide range of distribution and relatively small sample size, the reliability had been lowered. HR conducted from studies with a larger sample size had a range between 1.35 [21] and 2.25 [12]. 


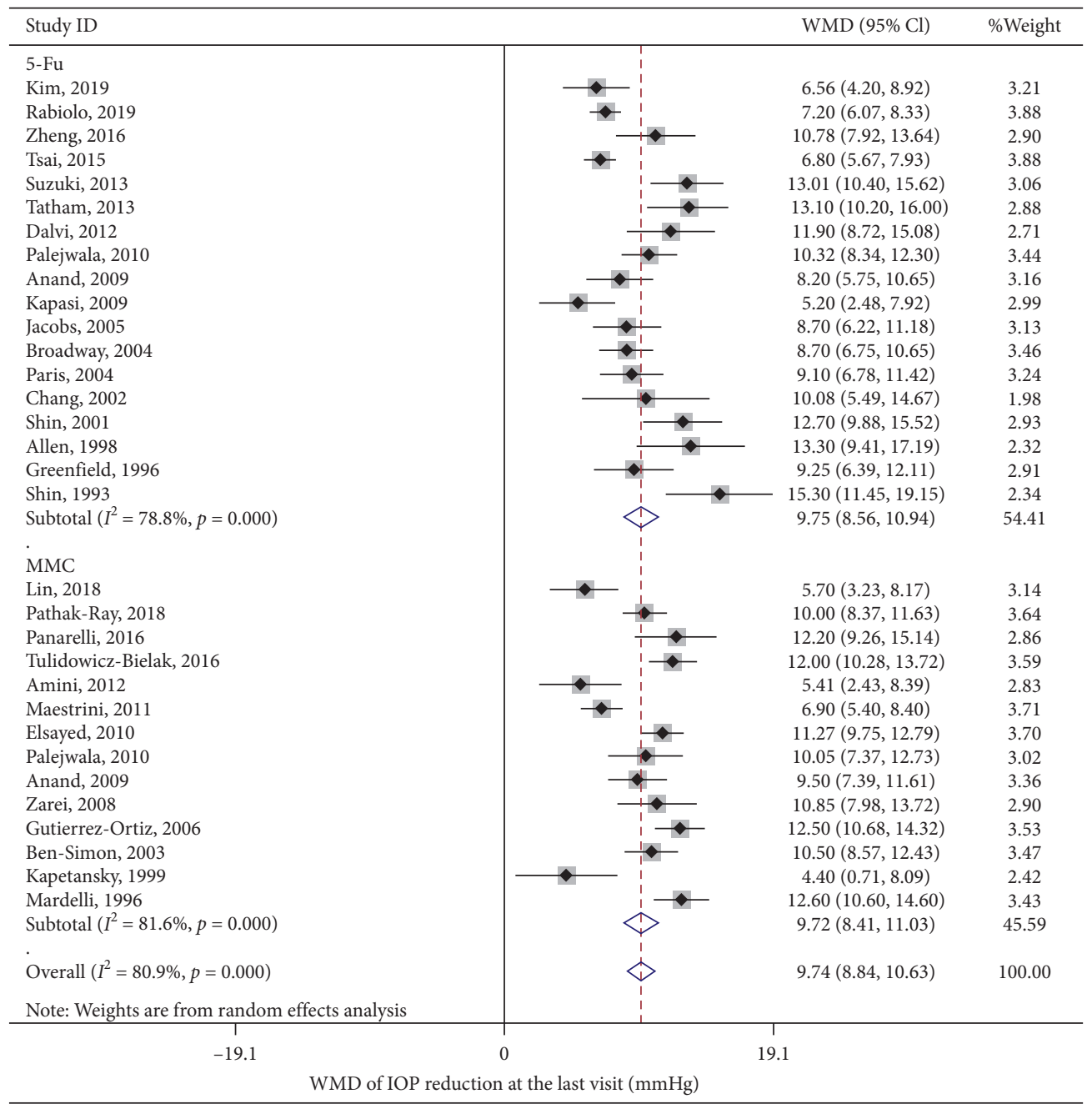

FIGURE 4: Weighted mean difference (WMD) of reduction in intraocular pressure (IOP) from baseline to the last visit. Subgroup analysis displayed the MMC and 5-Fu was used as an augmentation in bleb needling surgery.

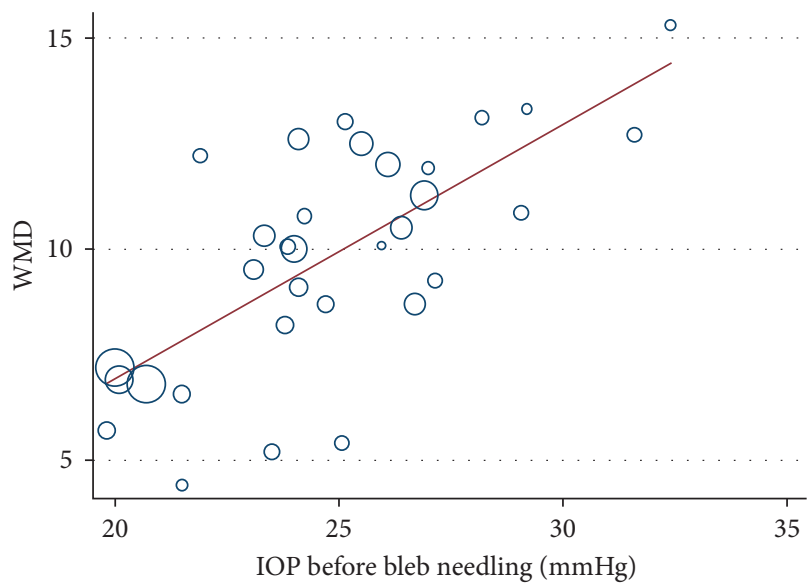

FIGURE 5: Metaregression for IOP before bleb needling: metaregression revealed that IOP before bleb needling significantly contributed to the heterogeneity of WMD of IOP reduction at last visit $(p<0.001)$.
IOP was another potential risk factor in surgery failure. IOP drop immediately after the surgery [29], typically with the cut-off value of $10 \mathrm{mmHg}[2,13,39]$, was a predictor for success. In comparison, among the eleven studies analyzed risk factors, only two studies showed that higher IOP before bleb needling was slightly more likely to cause a worse outcome $[10,21]$.

Timing selection for bleb needling after trabeculectomy was considered significant for surgical outcomes in two studies [2, 20]. Lee et al. [20] claimed that timing was the only significant risk factor in a Cox regression model. However, in another study, within three months after trabeculectomy, needling bleb can survive up to 12 months $(\mathrm{HR}=2.2)$ but did not reach a significant outcome in the long-term [2].

\section{Discussion}

4.1. Key Findings. We performed a meta-analysis of 37 studies that included patients who underwent bleb needling with subconjunctival injection of antimetabolites after 
TABLE 2: Estimates with 95\% confidence interval for complication rates after bleb needling.

\begin{tabular}{lcccccc}
\hline & \multicolumn{2}{c}{ Overall } & \multicolumn{2}{c}{ 5-Fu } & \multicolumn{2}{c}{ MMC } \\
& Estimate (\%) & 95\% CI & Estimate (\%) & 95\% CI & Estimate (\%) & 95\% CI \\
\hline Conjunctival haemorrhage & 5.7 & {$[2.5 \%, 10.1 \%]$} & 2.7 & {$[1.1 \%, 4.9 \%]$} & 10.8 & {$[2.9 \%, 22.9 \%]$} \\
Hyphema & 5.5 & {$[3.0 \%, 8.7 \%]$} & 3.9 & {$[1.8 \%, 6.8 \%]$} & 7.4 & {$[3.0 \%, 13.6 \%]$} \\
Bleb leakage & 5.0 & {$[3.2 \%, 7.3 \%]$} & 5.3 & {$[2.9 \%, 8.4 \%]$} & 4.8 & {$[2.1 \%, 8.4 \%]$} \\
Hypotony & 4.3 & {$[2.9 \%, 6.0 \%]$} & 3.6 & {$[1.9 \%, 5.9 \%]$} & 6.0 & {$[3.0 \%, 8.0 \%]$} \\
Shallow anterior chamber & 3.4 & {$[1.6 \%, 5.8 \%]$} & 1.7 & {$[0.6 \%, 3.4 \%]$} & 5.6 & {$[1.9 \%, 11.0 \%]$} \\
Serous choroidal detachment & 2.6 & {$[1.2 \%, 4.5 \%]$} & 1.5 & {$[0.6 \%, 2.7 \%]$} & 4.1 & {$[1.3 \%, 8.5 \%]$} \\
Corneal punctuate epitheliopathy & 2.4 & {$[1.1 \%, 4.2 \%]$} & 2.0 & {$[0.4 \%, 4.7 \%]$} & 2.9 & {$[1.1 \%, 5.6 \%]$} \\
Choroidal effusion & 1.3 & {$[0.6 \%, 2.1 \%]$} & 1.5 & {$[0.7 \%, 2.7 \%]$} & 1.0 & {$[0.2 \%, 2.3 \%]$} \\
\hline
\end{tabular}

${ }^{*}$ CI: confidence interval; MMC: mitomycin C; 5-Fu: 5-fluorouracil.

trabeculectomy failure. Our results showed that the bleb needling significantly lowered the IOP and reduced the number of antiglaucomatous medications. The overall effects of bleb needling with antimetabolites at the last visit revealed a reduction in IOP of $9.74 \mathrm{mmHg}, 45.9 \%$ for complete success rate, and $70.4 \%$ for qualified success rate. Despite similar efficacy profiles for MMC and 5-Fu, MMC showed higher estimate rates of complications. Baseline IOP was found to be a significant source of heterogeneity between the studies and had a positive correlation with the IOP reduction level.

4.2. Efficacy and Possible Rationale. Most of the studies we included evaluated the efficacy by success rate. However, as the definition of success or failure varied among studies, we additionally selected IOP value as a primary outcome because of its objectivity. Specifically, Paris et al. [34] and Kapetansky and Kapetansky [8] concentrated on IOP alterations rather than success rate. The reduction of IOP value was significant after bleb needling but revealed no apparent difference between the antimetabolites [19, 26, 29], which correlated with our subgroup analysis results. As antimetabolites were applied to keep the wound from fibrosis, a similar effect on IOP reduction further validated the efficacy of bleb needling itself. Although long-term studies had proved that the impact could sustain for more than three years but gradually decrease in success rate with time $[13,15,24,29]$, an overall analysis of the IOP reduction level had shown a stable trend in more than six months in our results. Therefore, we considered that the present metaanalysis provides generalizable information for bleb needling in clinical settings.

Our results had revealed that MMC application was slightly better than $5-\mathrm{Fu}$ in complete success rate but with higher complication rates, which corresponded to the potency of these medications. Direct comparison of MMC and 5-Fu application revealed that MMC outweighed 5-Fu in complete and qualified success rates with no significantly different complication rates $[19,29]$. One retrospective study claimed no apparent difference between the two groups [26]. A possible explanation was that the modified criteria of success rate depending on the reduction rate instead of the absolute value of IOP might influence the results [26]. When considering the mechanism, antimetabolites modulate wound healing based on different targets. Typically, 5-Fu was specific for fibroblasts, while MMC inhibits both fibroblasts and endothelial cells [45]. Given this mechanism, MMC exerts a more potent and durable effect than 5-Fu and requires fewer doses during clinical practice.

As for secondary outcomes, the reduction of IOP at six months, one year, and two years remained stable in our pooled results, indicating a long-lasting effect of IOP control. However, only 5 of 37 included studies had a mean followup of over three years $[13,18,24,29,39]$. As the results were not sufficient for aggregation, estimates for the long-term effects of bleb needling were less robust in our meta-analysis.

4.3. Safety Profile. With regard to safety, our estimates of procedure-related adverse events were evaluated as overall rates among all the studies. Subgroup analysis revealed that MMC might have higher complication rates than 5-Fu, which was also relevant to the higher antifibrosis power and longer duration of MMC [45]. A direct comparison between MMC and 5-Fu showed no significant difference in the complication in an RCT [19]. Possible reasons may include the avoidance of insertion under the scleral flap or into the anterior chamber, which significantly reduced the incidence of complications for both medications. Concerning the statistics of specific studies, two studies classified the complications by time, among which hyphema [25] and corneal punctate epitheliopathy [10], respectively, had the highest rate during the early stage. Differences might originate from time selection for bleb needling between the studies, which was related to the scar formation, and the antifibrosis power of adjunctive antimetabolites [45].

4.4. Risk Factors. The total number of needling procedures revealed a significant influence on surgical outcomes among studies $[2,12-14,21]$. A possible explanation was that more attempts for needling in an individual patient represented a worse control of IOP after the initial procedure $[2,12]$. Such a repetition of needling added the likelihood of poor prognosis. Specifically, for target IOP control, the number of needling tended to outweigh other factors with stricter criteria [13].

The predictive value of bleb morphology for surgical outcomes remained unclear $[2,7,8,13,17,20,35]$. Comparing the researches, the description of blebs varied greatly. 
A rough classification of bleb $[7,8,13,17]$ was more commonly used during evaluation, but its potential correlation with needling outcomes was weakened as it may ignore the severity of failed blebs. Though Than et al. [13] indicated the association of injected bleb and surgical success, the credibility was relatively low due to the subjective assessment for bleb morphology. A commonly-used Moorfields Bleb Grading System (MBGS) for bleb was therefore applied to evaluate the appearance [20]. Final success correlated with central bleb extension and height in this article. With this method, bleb morphology was comparable within different studies, yet it was not applied to the assessment for needling in most of the studies. Therefore, the relationship between bleb and needling outcomes remained to be explored.

As IOP and time selection were also considered predictive of surgical outcomes in different studies, risk factors for surgical failure defined as uncontrolled IOP did not reach a consensus among studies $[2,10,12-14,20,21,29,35,37,39]$.

4.5. Heterogeneity. Heterogeneity was a major concern for our meta-analysis. Many had mentioned that the studies differ in design, sample sizes, follow-up periods, criterion for success, and so on $[10,13,18]$, which made it difficult for comparison. Subgroup analysis in this study assessed the heterogeneity across the domains mentioned previously. Typically, regarding the bleb morphology before needling, a subgroup of three studies focusing on encapsulated bleb had lower heterogeneity than those not defined $[23,30,40]$. Considering the influence of a single study, Shin et al. [9] reported a maximum of $15.3 \mathrm{mmHg}$ of mean IOP reduction, and Kapetansky and Kapetansky [8] reported a minimum of $4.4 \mathrm{mmHg}$. Nevertheless, sensitivity analysis revealed that exclusions of any single study did not alter the pooled results, possibly due to their small sample size, which added robustness to our main findings. Further metaregression displayed a positive linear trend between IOP before bleb needling and WMD, strongly indicating a primary source of heterogeneity. After adjusting, the heterogeneity level turned into moderate for the meta-analysis results. Given that the variance of study settings was high, we displayed the surgical details for bleb needling in each study. Of note, differences in technique included the site of puncture, distance from the scleral flap, anaesthesia, concentration of antimetabolites, entry beneath the scleral flap, steroid application, and so on. Currently, limited studies had focused on these variables in bleb needling. We reasoned that the high heterogeneity also possibly originated from glaucoma subtype, proficiency of the ophthalmologists, bleb morphology, limbus/fornix based trabeculectomy, phakic status, antimetabolites application, or dosage of antimetabolites, which were hard to evaluate within studies.

4.6. Strengths and Limitations. As far as we know, limited systemic reviews had focused on bleb needling [6]. The study including 25 cases with encapsulated blebs found similar efficacy for bleb needling and medical treatment but claimed the priority of the latter [46]. The conclusion was drawn based on small sample sizes and under the circumstances of less widely application of antimetabolites in that era. However, with the introduction of antimetabolites, bleb needling had become a more common procedure after trabeculectomy failure. Moreover, bleb needling was not limited to encapsulation in real-world settings. A recent RCT included 40 eyes with encapsulated blebs found that bleb needling with antimetabolites had a lower mean IOP at 12 months [23]. Another long-term outcome revealed that needling with 5 -Fu for failing blebs had similar IOP control with those who had not undergone needling [24]. However, efficient as it was, bleb needling had displayed varied results due to diverse settings in a series of studies. For glaucoma specialists, the predictability of IOP reduction value and complication rates was crucial for treatment options. Our results were more practical with the inclusion of a larger sample and updated data for bleb needling with antimetabolites. Therefore, it can serve as a reference for treatment selection based on the estimations.

Several limitations of our study still need to be acknowledged. First, since a majority of studies were retrospective and noncomparative, variations in study design, methodology, and patient characteristics inevitably introduced relatively high heterogeneity, which we had discussed previously. Potential bias may lower the quality of our results. Second, the direct comparison of the antimetabolites was insufficient due to the original article setting we included. Although we observed the trends that MMC had slightly better IOP control than 5-Fu, the significance could not be told based on our pooled analysis. Third, criteria of success were modified in different studies based on their data. Those with a higher cut-off of IOP value and acceptance of repeating needling may potentially gain a higher success rate. As we chose a less strict definition, underestimation of success rate may be introduced during the pooled analysis. Moreover, neither short-term ( $<6$ months) nor long-term ( $>2$ years) efficacy of bleb needling was presented due to lack of data. Typically, owing to the probable loss of follow-up, long-term data with high quality may still be needed. Further direction for bleb needling researches may include long-term prospective study comparing the efficacy and safety for different adjuvant. The control group should be selected tactfully to increase the reliability of clinical judgment and treatment options in future studies.

\section{Conclusion}

In conclusion, bleb needling with antimetabolites could be considered an effective and safe procedure after trabeculectomy failure. After the process, patients will gain ideal IOP control and reduce antiglaucomatous medications for at least six months with 5-Fu or MMC. Meanwhile, the overall estimates for complications were relatively low in the whole process. An increasing number of needling was the leading risk factor for needling failure.

\section{Data Availability}

The pooled analysis data used in this study are available from the corresponding author upon request. 


\section{Conflicts of Interest}

The authors declare that there are no conflicts of interest regarding the publication of this article.

\section{Authors' Contributions}

$\mathrm{YH}$ and $\mathrm{CZ}$ were responsible of conceptualization. XC and $\mathrm{YH}$ were responsible of data extraction and formal analysis. $\mathrm{XC}$ was responsible of software. $\mathrm{YH}$ and $\mathrm{CZ}$ were responsible of supervision. XC was responsible of original drafting. LS, YH, and CZ were responsible of writing, review, and editing. XC, LS, YH, and CZ were responsible of approval of the final version:. All authors read and approved the final manuscript.

\section{Acknowledgments}

This work was supported by Capital's Funds for Health Improvement and Research (no. CFH-2020-2-40911).

\section{Supplementary Materials}

The search strategy was included in the supplementary file. Supplementary Figure 1: the risk of bias graph for included studies. Supplementary Figures 2-6: the funnel plots for WMD of IOP reduction at the different timeline and changes in antiglaucomatous medications. Supplementary Figures 7-10: the forest plots for WMD of IOP reduction at the different timeline and changes in antiglaucomatous medications. Supplementary Figure 11: the sensitivity analysis for WMD of IOP reduction at the last visit. Supplementary Table 1: the detailed technique of bleb needling in each study. Supplementary Table 2: subgroup analysis results for WMD of IOP reduction at the last visit. Supplementary Table 3: metaregression results for WMD of IOP reduction at the last visit. (Supplementary Materials)

\section{References}

[1] J. Landers, K. Martin, N. Sarkies, R. Bourne, and P. Watson, "A twenty-year follow-up study of trabeculectomy: risk factors and outcomes," Ophthalmology, vol. 119, no. 4, pp. 694-702, 2012.

[2] A. P. Rotchford and A. J. W. King, "Needling revision of trabeculectomies," Ophthalmology, vol. 115, no. 7, pp. 1148-1153.E4, 2008.

[3] R. H. Ewing and R. L. Stamper, "Needle revision with and without 5-fluorouracil for the treatment of failed filtering blebs," American Journal of Ophthalmology, vol. 110, no. 3, pp. 254-259, 1990.

[4] P. G. Mardelli, C. M. Lederer ., P. L. Murray, S. A. Pastor, and K. M. Hassanein, "Slit-lamp needle revision of failed filtering blebs using mitomycin C," Ophthalmology, vol. 103, no. 11, pp. 1946-1955, 1996.

[5] K. Mercieca, B. Drury, A. Bhargava, and C. Fenerty, "Trabeculectomy bleb needling and antimetabolite administration practices in the UK: a glaucoma specialist national survey," British Journal of Ophthalmology, vol. 102, no. 9, pp. 12441247, 2018.
[6] A. Feyi-Waboso and H. O. Ejere, "Needling for encapsulated trabeculectomy filtering blebs," Cochrane Database of Systematic Reviews, vol. 8, Article ID Cd003658, 2012.

[7] H. Amini, A. Esmaili, R. Zarei, N. Amini, and R. Daneshvar, "Office-based slit-lamp needle revision with adjunctive mitomycin-C for late failed or encapsulated filtering blebs," Middle East African Journal of Ophthalmology, vol. 19, no. 2, pp. 216-221, 2012.

[8] F. M. Kapetansky and S. D. Kapetansky, "Antimetabolite use in revising failing filtering blebs," Seminars in Ophthalmology, vol. 14, no. 3, pp. 144-151, 1999.

[9] D. H. Shin, M. S. Juzych, A. K. Khatana, R. P. Swendris, and K. A. Parrow, "Needling revision of failed filtering blebs with adjunctive 5-fluorouracil," Ophthalmic Surgery, vol. 24, no. 4, pp. 242-248, 1993.

[10] A. Rabiolo, A. Marchese, P. Bettin et al., "Needle revision outcomes after glaucoma filtering surgery: survival analysis and predictive factors," European Journal of Ophthalmology, vol. 30, no. 2, pp. 350-359, 2020.

[11] M. Okka, E. Mirza, R. Oltulu, S. Belviranlı, and M. K. Gündüz, "The efficacy of bleb needling revision with 5fluorouracil in encapsulated bleb after unsuccessful trabeculectomy," The European Research Journal, vol. 5, no. 6, pp. 990-995, 2019.

[12] J.-S. Kim, H. J. Kim, K. I. Na, Y. K. Kim, K. H. Park, and J. W. Jeoung, "Comparison of efficacy and safety of bleb needle revision with and without 5-fluorouracil for failing trabeculectomy bleb," Journal of Glaucoma, vol. 28, no. 5, pp. 386-391, 2019.

[13] J. Y. X. L. Than, T. S. Al-Mugheiry, J. Gale, and K. R. Martin, "Factors predicting the success of trabeculectomy bleb enhancement with needling," British Journal of Ophthalmology, vol. 102, no. 12, pp. 1667-1671, 2018.

[14] V. Pathak-Ray and N. Choudhari, "Rescue of failing or failed trabeculectomy blebs with slit-lamp needling and adjunctive mitomycin C in Indian eyes," Indian Journal of Ophthalmology, vol. 66, no. 1, pp. 71-76, 2018.

[15] S. Lin, D. Byles, and M. Smith, "Long-term outcome of mitomycin C-augmented needle revision of trabeculectomy blebs for late trabeculectomy failure," Eye, vol. 32, no. 12, pp. 1893-1899, 2018.

[16] L. Zheng, H. Arvind, and D. Wechsler, “Outcomes," Journal of Glaucoma, vol. 25, no. 3, pp. 317-323, 2016.

[17] M. Tulidowicz-Bielak, E. Kosior-Jarecka, and T. Żarnowski, "Revision of trabeculectomy filtering blebs with mitomycin C: long term results," Indian Journal of Ophthalmology, vol. 64, no. 11, pp. 822-828, 2016.

[18] J. F. Panarelli, K. Vinod, G. Huang, and P. A. Sidoti, "Transconjunctival revision with mitomycin-C following failed trabeculectomy," Journal of Glaucoma, vol. 25, no. 7, pp. 618-622, 2016.

[19] W. Liu, J. Wang, M. Zhang, Y. Tao, and Y. Sun, "Comparison of subconjunctival mitomycin $\mathrm{C}$ and 5-fluorouracil injection for needle revision of early failed trabeculectomy blebs," Journal of Ophthalmology, vol. 2016, Article ID 3762674, 6 pages, 2016.

[20] Y. S. Lee, S. C. Wu, H. J. Tseng, W. C. Wu, and S. H. Chang, "The relationship of bleb morphology and the outcome of needle revision with 5-fluorouracil in failing filtering bleb," Medicine, vol. 95, no. 36, Article ID e4546, 2016.

[21] A. S. Tsai, P. Y. Boey, H. M. Htoon, and T. T. Wong, "Bleb needling outcomes for failed trabeculectomy blebs in Asian eyes: a 2-year follow up," International Journal of Ophthalmology, vol. 8, no. 4, pp. 748-753, 2015. 
[22] A. Tatham, U. Sarodia, and W. Karwatowski, "5-Fluorouracil augmented needle revision of trabeculectomy," Journal of Glaucoma, vol. 22, no. 6, pp. 463-467, 2013.

[23] R. Suzuki and R. Susanna-Jr, "Early transconjunctival needling revision with 5-fluorouracil versus medical treatment in encapsulated blebs: a 12-month prospective study," Clinics, vol. 68, no. 10, pp. 1376-1379, 2013.

[24] R. Dalvi, N. Orzech, C. Kranemann, and C. M. Birt, "Five-year results of 5-fluorouracil augmented needling revision of failing blebs," Ophthalmic Surgery, Lasers, and Imaging, vol. 43, no. 1, pp. 32-38, 2012.

[25] H. A. Maestrini, S. Cronemberger, H. D. S. Matoso et al., "Late needling of flat filtering blebs with adjunctive mitomycin C: efficacy and safety for the corneal endothelium," Ophthalmology, vol. 118, no. 4, pp. 755-762, 2011.

[26] N. Palejwala, P. Ichhpujani, G. Fakhraie, J. S. Myers, M. R. Moster, and L. J. Katz, "Single needle revision of failing filtration blebs: a retrospective comparative case series with 5fluorouracil and mitomycin C," European Journal of Ophthalmology, vol. 20, no. 6, pp. 1026-1034, 2010.

[27] T. H. Elsayed and T. M. El-Raggal, "Mitomycin-C needle bleb revision in congenital glaucoma," Middle East African Journal of Ophthalmology, vol. 17, no. 4, pp. 369-373, 2010.

[28] M. S. Kapasi and C. M. Birt, "The efficacy of 5-fluorouracil bleb needling performed 1 year or more posttrabeculectomy," Journal of Glaucoma, vol. 18, no. 2, pp. 144-148, 2009.

[29] N. Anand and A. Khan, "Long-term outcomes of needle revision of trabeculectomy blebs with mitomycin $\mathrm{C}$ and 5fluorouracil," Journal of Glaucoma, vol. 18, no. 7, pp. 513-520, 2009.

[30] R. Zarei, S. Shahhosseini, G. Faragee-Oskouee, A. R. Shokoh, and K. Hamzehdoost, "Needle revision with mitomycin-C in encapsulated blebs," Acta Medica Iranica, vol. 46, no. 4, pp. 295-298, 2008.

[31] C. Gutierrez-Ortiz, C. Cabarga, and M. A. Teus, "Prospective evaluation of preoperative factors associated with successful mitomycin C needling of failed filtration blebs," Journal of Glaucoma, vol. 15, no. 2, pp. 98-102, 2006.

[32] R. K. Shetty, L. Wartluft, and M. R. Moster, "Slit-lamp needle revision of failed filtering blebs using high-dose mitomycin C," Journal of Glaucoma, vol. 14, no. 1, pp. 52-56, 2005.

[33] S. Jacobs, A. Gillis, L. Van Malderen, and T. Zeyen, "Needlingrevision of failed filtering blebs," Bulletin de la Societe Belge d'Ophtalmologie, vol. 297, pp. 59-64, 2005.

[34] G. Paris, M. Zhao, and W. E. Sponsel, "Operative revision of non-functioning filtering blebs with 5-fluorouracil to regain intraocular pressure control," Clinical and Experimental Ophthalmology, vol. 32, no. 4, pp. 378-382, 2004.

[35] D. Broadway, P. A. Bloom, C. Bunce, M. Thiagarajan, and P. T. Khaw, "Needle revision of failing and failed trabeculectomy blebs with adjunctive 5-fluorouracil: survival analysis," Ophthalmology, vol. 111, no. 4, pp. 665-673, 2004.

[36] G. J. Ben-Simon and Y. Glovinsky, "Needle revision of failed filtering blebs augmented with subconjunctival injection of mitomycin C," Ophthalmic Surgery, Lasers and Imaging Retina, vol. 34, no. 2, pp. 94-99, 2003.

[37] A. S. Hawkins, J. K. Flanagan, and S. V. L. Brown, "Predictors for success of needle revision of failing filtration blebs," Ophthalmology, vol. 109, no. 4, pp. 781-785, 2002.

[38] S. H. Chang and C. H. Hou, "Needling revision with subconjunctival 5-fluorouracil in failing filtering blebs," Chang Gung Medical Journal, vol. 25, no. 2, pp. 97-103, 2002.

[39] D. H. Shin, Y. Y. Kim, S. Y. Ginde et al., "Risk factors for failure of 5-fluorouracil needling revision for failed conjunctival filtration blebs," American Journal of Ophthalmology, vol. 132, no. 6, pp. 875-880, 2001.

[40] L. E. Allen, K. Manuchehri, and P. G. Corridan, "The treatment of encapsulated trabeculectomy blebs in an out-patient setting using a needling technique and subconjunctival 5fluorouracil injection," Eye, vol. 12, no. Pt 1, pp. 119-123, 1998.

[41] D. S. Greenfield, M. P. Miller, I. J. Suner, and P. F. Palmberg, "Needle elevation of the scleral flap for failing filtration blebs after trabeculectomy with mitomycin C," American Journal of Ophthalmology, vol. 122, no. 2, pp. 195-204, 1996.

[42] A. Liberati, D. G. Altman, J. Tetzlaff et al., "The PRISMA statement for reporting systematic reviews and meta-analyses of studies that evaluate healthcare interventions: explanation and elaboration," The British Medical Journal, vol. 339, Article ID b2700, 2009.

[43] M. Viswanathan, M. T. Ansari, N. D. Berkman et al., "AHRQ methods for effective Health care: assessing the risk of bias of individual studies in systematic reviews of Health care interventions," in Methods Guide for Effectiveness and Comparative Effectiveness ReviewsAgency for Healthcare Research and Quality (US), Rockville MD, USA, 2008.

[44] S. P. Hozo, B. Djulbegovic, and I. Hozo, "Estimating the mean and variance from the median, range, and the size of a sample," BMC Medical Research Methodology, vol. 5, p. 13, 2005.

[45] L. K. Seibold, M. B. Sherwood, and M. Y. Kahook, "Wound modulation after filtration surgery," Survey of Ophthalmology, vol. 57, no. 6, pp. 530-550, 2012.

[46] V. P. Costa, M. M. Correa, and N. Kara-Jose, "Needling versus medical treatment in encapsulated blebs," Ophthalmology, vol. 104, no. 8, pp. 1215-1220, 1997. 